Introduction: The earthquake-resistant standard of the buildings of Japan is maintained by several levels. After the Great Hanshin-Awaji Earthquake(1995) the Ministry of Land, Infrastructure, Transport, and Tourism in Japan classified the earthquake-resistant performance for the base facilities into 3 levels. The hospital manager often selects the middle level of earthquake-resistance. However, 10 hospitals were closed down for the destruction of facilities by the Kumamoto earthquake. Who may evaluate the safety of a hospital after a great earthquake? The purpose of this study is to consider the methods to evaluate the safety of hospital buildings just after a great earthquake.

Methods: The damage to hospitals and the measures based on Japanese Law are arranged. Then it is considered who can declare the safety of hospital buildings after a great earthquake. Results: Hospital buildings collapsed in the Hanshin-Awaji Great Earthquake and many hospitals lost a function by a tsunami in the Great East Japan Earthquake. In addition, the glass and the ceilings of the hospital were damaged in the Kumamoto Earthquake. The damage occurred although these many hospitals had an earthquake-resistant standard established in the Building Standard Act of Japan. It is necessary for the experts to judge the safety of the hospital building just after a great earthquake.

Discussion: The safety of hospital buildings is the responsibility of the hospital manager. However, there isn't an expert of building structure employed as staff at a hospital. Thus, the hospital personnel must allow the expert of the building structure to advise a manager. In the future, it is important that the evaluation methods that can judge the damage of a hospital are developed, and the practical training for the hospital personnel are repeated.

Prehosp Disaster Med 2019;34(Suppl. 1):s177-s178

doi:10.1017/S1049023X19004084

\section{A Verification Test for an Assessment System: Rapid Assessment System of Evacuation Center Condition- Gonryo and Miyagi (RASECC-GM) that Utilizes Mobile Devices \\ Prof. Tadashi Ishii ${ }^{1}$, Dr. Kazuma Morino ${ }^{2}$, Prof. Masaharu Nakayama ${ }^{3}$, Prof. Tomohiko Mase ${ }^{4}$, Assistant Prof. Hiroyuki Fujiwara ${ }^{4}$, Assistant Prof. Manabu Icbikawa \\ 1. Tohoku University Hospital, Sendai, Japan \\ 2. Yamagata Prefectural Central Hospital, Yamagata-shi, Japan \\ 3. Tohoku University School of Medicine, Sendai-shi, Japan \\ 4. Iwate Medical University, Shiwa-gun, Japan \\ 5. Shibaura Institute of Technology, Saitama-shi, Japan}

Introduction: On March 11, 2011, the Great East Japan Earthquake struck the northeastern coast of Japan with the magnitude nine. Ishinomaki medical zone was affected most severely with 328 evacuation shelters and approximately 50,000 evacuees. The Ishinomaki Zone Joint Relief Team gathered information directly from all evacuation shelters using assessment sheets. Based on this assessment data, various measures were carried out for environmental improvement of the shelters. To prepare for the next major disaster, a software program called Rapid Assessment System of Evacuation Center Condition - Gonryo and Miyagi (RASECC-GM) was developed, which computerizes the whole process, including entering, tabulating, and managing of shelter assessment data. Aim: To verify the feasibility, usability, and accuracy of RASECC-GM, a verification test was performed using mock shelter data on October 23-26, 2018, to coincide with Logistics Training Course of Medical Logistics for Disasters held by Iwate Medical University.

Methods: On October 22, 2018 at four simulated disaster relief and health care branches, participants at each branch were asked to enter two mock shelter data items, submit a closed shelter request, and register a new shelter using RASECC-GM, respectively. The next day participants were asked to enter two mock shelter data items per branch while offline and upload the data to the server when next online. The uploaded data was checked for accuracy and whether it could be viewed on the management screen. After the test, a questionnaire survey was given to participants to verify the feasibility and usability of RASECC-GM.

Results: It was confirmed that RASECC-GM functioned almost correctly. All participants answered that input operation was easy to understand, and $90.9 \%$ of participants could input without a mistake and did not feel stress when inputting data. Discussion: RASECC-GM appeared to be useful to shelter assessment, but further improvements are needed for practical use.

Prehosp Disaster Med 2019;34(Suppl. 1):s178

doi:10.1017/S1049023X19004096

\section{Who Revisits Medical Services at a Music Festival? \\ Dr. Matthew Brendan Munn ${ }^{1}$, Stefan Gogaert ${ }^{2}$ \\ 1. UBC Emergency Medicine, Vancouver, Canada \\ 2. Belgian Red Cross, Flanders, Belgium}

Introduction: Attendees at music festivals rely upon on-site medical services for their emergency and medical care needs. Patients previously cared for can re-present for services at different times over the course of an event.

Aim: To identify the proportion of visits that are repeat presentations at music festivals and discuss themes in the medical care needs of these potentially resource-intensive patients.

Methods: This study included a review of prospectively enrolled patients presenting for health services over five years at a number of music festivals in Belgium and Canada. Patient data were extracted from existing databases of visits as well as visit documentation, and linked by name and date of birth to identify repeat visits. Data were de-identified and visit times, triage acuity, chief complaints, treatments, and discharge instructions were extracted.

Results: Re-presentations constituted approximately $5 \%$ of all on-site medical visits. The majority were for minor care (e.g., wounds, dressings, foot care). Repeat visits for major issues included chronic disease (e.g., asthma, seizures, diabetes) and serial intoxications; these were high risk for transport to hospital. Festival duration was positively correlated with the number of patients with multiple visits. Three or more visits or visits in different years were rare occurrences.

Discussion: At music festivals, a small but significant proportion of attendees utilize medical services repeatedly. Most are 